\title{
Ramgen Rotor Cartridge for the Pre-Prototype Ramgen Engine Topical Report
}

October 1, 1999 to September 30, 2001

Principle Author: Ramgen Power Systems

Report Issued September 2003

DOE Award Number: DE-FC26-00NT40915

Document Number 0800-00070

Submitted by:

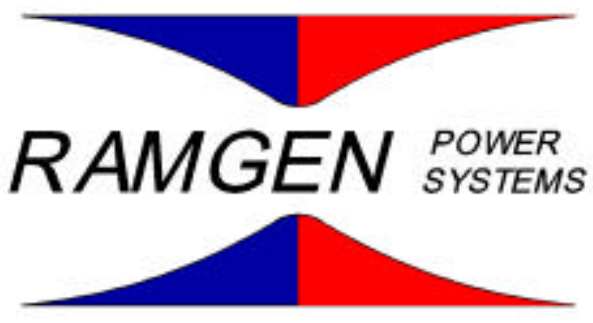

Ramgen Power Systems, Inc. 11808 Northup Way, Suite W190

Bellevue, WA 98005

(425) 828-4919 


\section{Disclaimer}

This report was prepared as an account of work sponsored by an agency of the United States Government. Neither the United States Government nor any agency thereof, nor any of their employees, makes any warranty, express or implied, or assumes any legal liability or responsibility for the accuracy, completeness, or usefulness of any information, apparatus, product, or process disclosed, or represents that its use would not infringe privately owned rights. Reference herein to any specific commercial product, process, or service by trade name, trademark, manufacturer, or otherwise does not necessarily constitute or imply its endorsement, recommendation, or favoring by the United States Government or any agency thereof. The views and opinions of authors expressed herein do not necessarily state or reflect those of the United States Government or any agency thereof. 


\begin{abstract}
The research and development of a unique combustion engine is presented. The engine converts the thrust from ramjet modules located on the rim of a disk into shaft torque, which in turn can be used for electrical power generation or mechanical drive applications. A test program was undertaken that included evaluation of the pre-prototype engine and incorporation of improvements to the thrust modules and supporting systems. Fuel mixing studies with vortex generators and bluff body flame holders demonstrated the importance of increasing the shearlayer area and spreading angle to augment flame volume. Evaluation of flame-holding configurations (with variable fuel injection methods) concluded that the heat release zone, and therefore combustion efficiency, could be manipulated by judicious selection of bluff body geometry, and is less influenced by fuel injection distribution. Finally, successful operation of novel fuel and cooling air delivery systems have resolved issues of gas (fuel and air) delivery to the individual rotor segments. The lessons learned from the pre-prototype engine are currently being applied to the development of a 2.8MW engine. Keywords: Gas Turbine, Ramjets, LeanPremixed, Combustion.
\end{abstract}

This effort was funded in part by the Department of Energy under the cooperative agreement \#DE-FC26-00NT40, awarded between the period of September 29, 2000 and August 31, 2001. The computational support (Star CD) was provided by Combustion Science and Engineering (Columbia, MD). 


\section{Table of Contents}

1.0 Executive Summary

2.0 Introduction

3.0 Theory of Operation: The Ramgen Engine

3.1 Ramjet Technology

3.2 The Ramgen Engine

3.3 Experimental Results: The Pre-Prototype Engine

3.3.1 Experimental Set-up

3.3.1.1 Engine Layout

3.3.1.2 Flame Stabilizers

3.3.2 Experimental Results

3.3.2.1 Typical Test Sequence

3.3.2.2 Comparisons in Flame Stabilization Configurations

4.0 Conclusions

5.0 References 


\subsection{Executive Summary}

This report summarizes the results of Ramgen Engine development efforts.

The Ramgen engine represents the unique application of ramjet principles to power generation. The engine is designed to convert the thrust from a ramjet thrust module into shaft torque, which in turn can drive a generator to provide electricity of shaft power for a mechanical drive application. This effort was funded by Department of Energy cooperative agreement number DE-FC26-00NT40915.

The primary objective of advancing the research and development effort on the Ramgen engine was accomplished by reaching significant technological milestones. Major tasks completed include:

- Completion of an intensive test program, with over 500 cold flow, ignition, ramp, and high-speed engine tests. This included 216 hours of engine spin tests, 42.5 hours of hot combustor testing, and 6.3 hours above Mach 1.

- Testing three fuel mixing configurations using Vortex Generators.

- Design and testing a bluff body flame holder with integral fuel injection.

- Testing five pilot-fuel injection methods which incorporated rapid configuration change features.

- Ignition, ramping and steady state operation using methane (natural gas) and hydrogen fuels.

Accomplishment of these tasks has led to a greater understanding of what is required to adapt the ramjet technology to a rotating reference frame. Fuel mixing studies with Vortex Generators and a bluff body flame holder illuminated the importance of increasing the shear-layer spreading angle and maximizing the shear area, thereby augmenting flame surface area. Evaluation of flame-holding configurations (with variable fuel injection methods) concluded that the heat release zone, and therefore combustion efficiency, could be manipulated by judicious selection of bluff body geometry, and is less influenced by fuel injection distribution.

Based on the efforts to date, it is clear that optimizing combustor residence time and velocities while dealing with unique boundary conditions is of fundamental importance. Constraints on practical flame-holder design, emissions, thrust segment weight and geometry, and materials make optimization a worthy challenge. Significant milestones toward answering these challenges have been made in the contract period. Future efforts will build on the foundation of this research and development effort. 


\subsection{Introduction}

The paper presents the design and development of a fundamentally new kind of power generation device known as the Ramgen engine. These efforts have been aided by the Department of Energy, as part of an initiative to bring viable and innovative energy technologies to market.

The design of the pre-prototype engine represents the application of well-established ramjet principles to power generation. Instead of using a ramjet to provide thrust for a supersonic aircraft or missile, the engine converts the linear thrust from a ramjet into shaft torque, which in turn drives a generator which provides electricity or shaft power for a mechanical drive application. At maturity, the production engine will have a thermal efficiency rivaling and surpassing conventional gas turbines, with highly competitive $\mathrm{CO}$ and $\mathrm{NO}_{\mathrm{x}}$ emissions through its operation in a lean premixed mode. The unique engine design also permits the use of waste fuels, including landfill gas and coal bed methane as well as synthetic ("Syn Gas") and biomass fuels, for energy production. Such atypical gaseous fuels cannot be currently utilized in conventional gas turbine systems due to the risks of autoignition, flashback, engine wear and inability to ingest atmospheric fuels directly.

The pre-prototype engine was fully assembled in Tacoma, WA in July 1998. Tests occurring in 1999 validated ignition and flame holding at idle rotor speeds, the mechanical integrity of the rotor at supersonic tip speeds, and overall system integrity. Development work conducted between late 1999 to September 2000 focused on improving combustion stability, fuel delivery methods, and air film cooling systems. These efforts resulted in substantial progress toward operation at full rotor speeds (4300 rpm, Mach 1.1 inflow conditions). 


\subsection{Theory of Operation: The Ramgen Engine}

\subsection{Ramjet Technology}

The engine is based on ramjet technology which produces propulsive force by increasing the momentum of the working fluid via combustion and expansion through a supersonic nozzle (Heiser and Pratt, 1994). In contrast to other air-breathing engines, the working cycle of the ramjet engine is not accompanied by mechanical compression of the working fluid but rather through a shock system located within the inlet portion of the engine. This makes the ramjet engine the simplest mechanical air-breathing engine to date through its minimal use of moving parts. Figure 1 illustrates the working cycle of the ramjet engine and demonstrates the four fundamental processes: ingestion, compression, heat release, and expansion.

Flame stabilization is typically achieved from a simple flame holder (V-Gutter or equivalent) located at the end of the inlet passage or dump plane and sized for the appropriate flow velocities. The air-fuel mixture entering the combustion chamber is ignited by recirculating hot combustion products comprising the recirculation zone.

The flow within the combustor is subsonic; hence, an initial contraction of the flow area (throat) is necessary to both reduce the internal pressure and accelerate the flow to sonic conditions (Oates, 1997). After attaining sonic velocities, the flow encounters a diverging passage to continue the acceleration process. The addition of the nozzle allows the flow to continue to expand to supersonic exit velocities, until ambient pressure is attained - "ideal expansion".

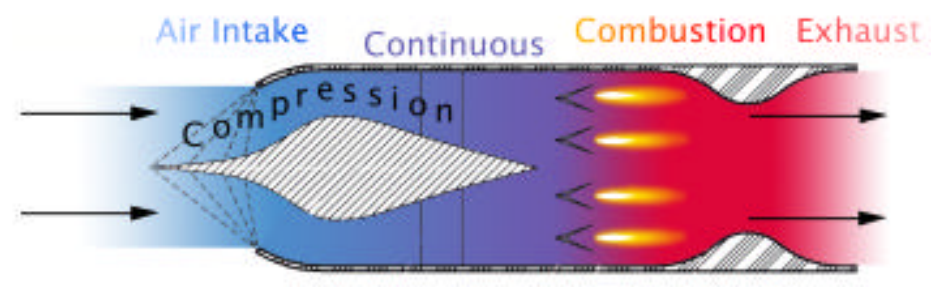

Fig. 1: Ramjet Engine Working Cycle.

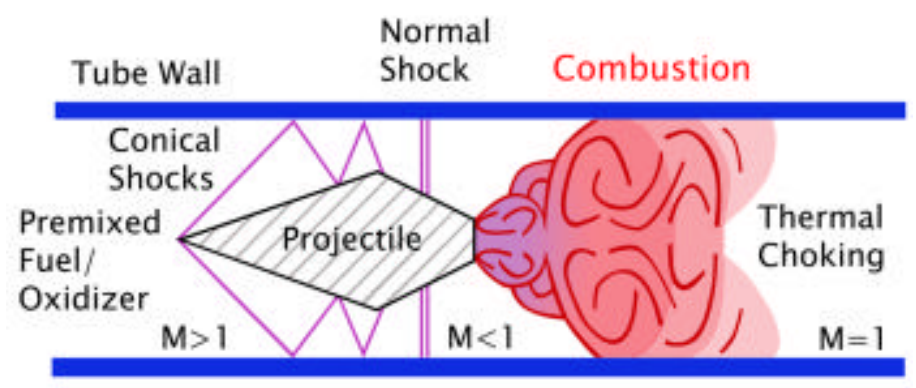

Fig. 2: The Ram Accelerator. 
A novel variation of the ramjet engine is the ram-accelerator. The ram-accelerator is a unique embodiment of the ramjet process applied to the acceleration of a projectile or payload (Hertzberg et al., 1988, Bogdanoff, 1992). In the ram-accelerator, a tube or barrel is filled with a mixture of fuel and oxidant, commonly referred to as "premix". A projectile is first launched into one end of the barrel with a low supersonic velocity (Mach 1.2). As the projectile travels through the premix, the shaping of the projectile creates a series of oblique, conical shock waves, which coalesce and reflect off the barrel walls as indicated in Fig. 2 (Bogdanoff, 1992, Harsha, 1981).

A normal shock is stabilized in the expanding section of the flowpath formed between the projectile and the barrel wall (see Fig. 2). As the premix passes through the normal shock wave and diffuser, it is compressed and decelerated to subsonic velocities. The combustion process is stabilized behind the trailing edge of the projectile - which acts as a rearward facing step flameholder - where after a thermally choking process ensues to maximize exit gas velocity. The duct or flow path formed between the moving projectile and the stationary barrel wall completes the ramjet's flowpath where all the thrust is manifested on the moving projectile. The thrust developed by the ramjet process accelerates the projectile as it moves along the length of the barrel so that its exit velocity greatly exceeds its inlet velocity.

\subsection{The Ramgen Engine}

The essential concept of the engine is the incorporation of one or more ramjets onto the rim of a rotor such that the thrust from the ramjets acts tangentially, causing it to rotate at supersonic rim speeds. In this way, the thrust from the ramjets is converted into shaft torque.

Figure 3 is an isometric illustration of the high-speed rotor and the stationary main engine case from the pre-prototype engine. The figure illustrates how the two ramjet flow-paths are incorporated into the rim of the high-speed rotor. As shown, the two-dimensional ramjet flowpaths are open on their exterior, or radially outer-most, surface. Closure of the ramjet flowpath is facilitated by the stationary surface of the main engine case. The combination of a ramjet flowpath where one of the ramjet surfaces is moving relative to the other is analogous to the moving projectile and stationary barrel wall of the ram-accelerator. The ramjet flow-paths are mounted on the rim of the rotor as inter-twined helixes at an optimized angle to ensure sufficient inlet length, combustor velocities, residence times and optimized thrust vectors. Combustion products from one ramjet are inhibited from being ingested into its neighbor by a mechanical separation known as a strake which wraps around the rotor's periphery (Fig. 3). Note that the helix/strake angle dictates the inlet, diffuser, combustor, and nozzle lengths, which in turn dictates the combustor velocity, residence time, and thrust vectors.

The shallow strake angle has a number of implications. Firstly, despite the supersonic rim speeds, the axial velocity of the premix ingested is very low. As a result, the inflow can be delivered to the ramjets with minimal pressure and viscous losses, facilitating a simple atmospheric premixer system. As a consequence, the engine is capable of burning a wide variety of low pressure and sub-quality fuels, including coal-bed methane, low-BTU natural gas (landfill methane), biomass fuels, and hydrogen. Secondly, the "auguring" nature of the rotor enables the 
engine to be self-pumping, drawing in the required flow as determined by the effective flow area of the inlet system.

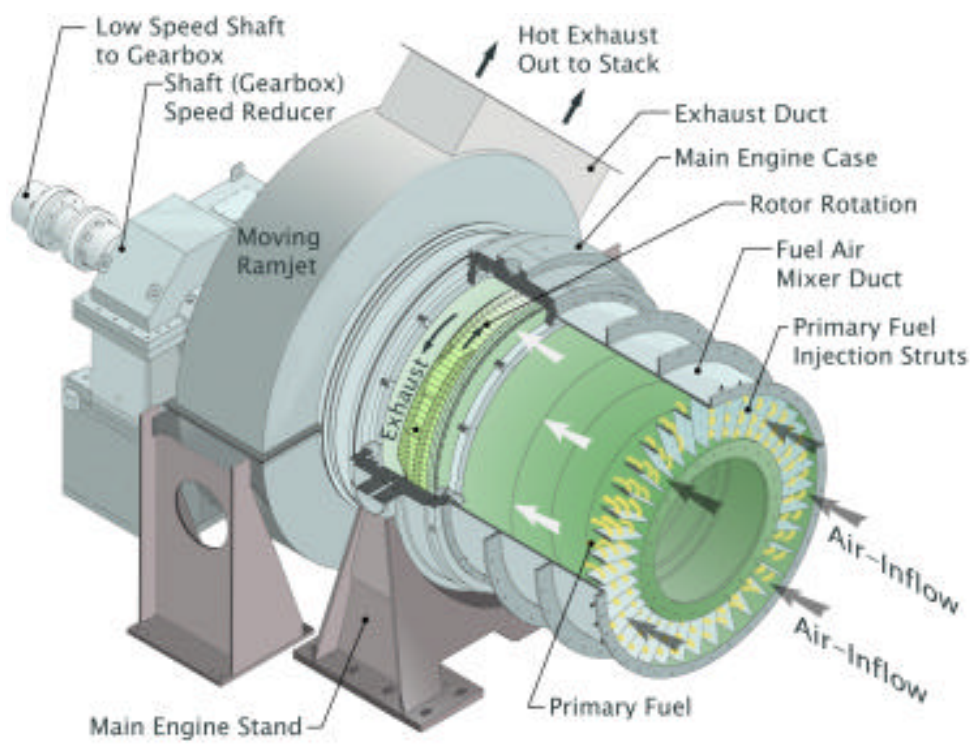

Fig. 3: The Pre-Prototype Ramgen Engine.

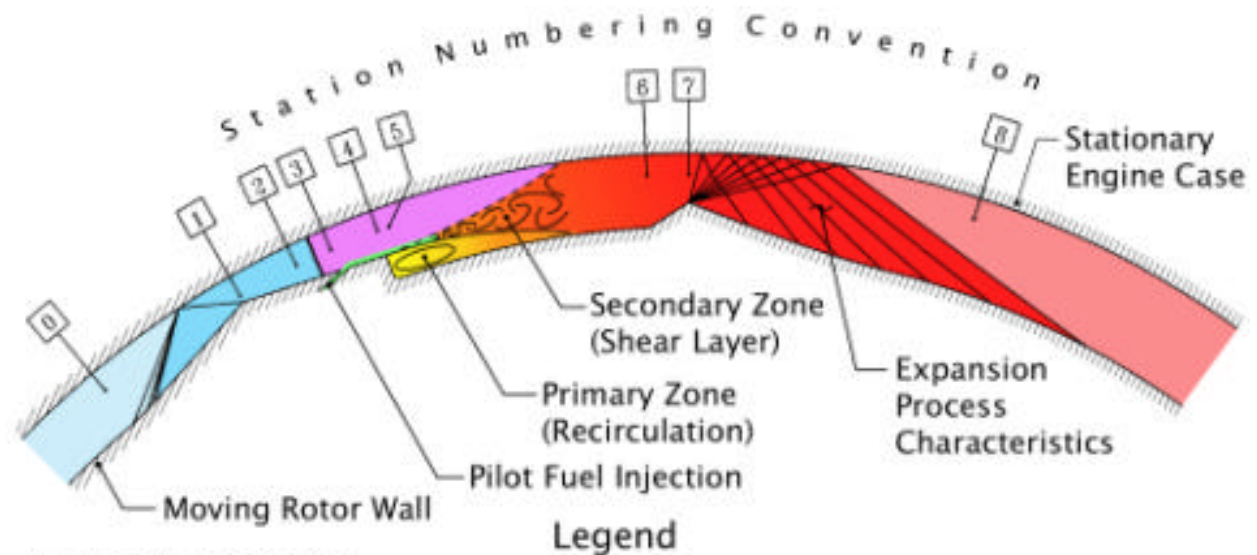

Station 0 - Inlet Flow

Station 1 - Post Oblique Shock

Station 5 - Combustor Inflow

Station 2 - Pre Normal Shock

Station 3 - Post Normal Shock

Station 6 - Post Combustion

Station 7 - Nozzle Throat

Station 4 - Complete Subsonic Expansion Station 8 - Complete Expansion

Fig. 4: Ramgen Engine Flow Details.

Figure 4 is a cut-away view depicting the flow path including the supersonic inlet, diffuser, combustor and exit nozzle for the pre-prototype engine. As shown in the figure, supersonic inflow (Station 0) meets the compression surface comprising the inlet. Through a series of Mach waves and strongly reflected shock (emanating from the stationary casing wall); the required 
inlet compression is achieved. The gas is then diffused through a two dimensional diffuser whereby the static pressure rises to attain required combustor inflow conditions while ensuring no separation along the diffuser's length. A simple rearward facing step flameholder is employed as the baseline flameholder to anchor the combustion activity. Standard diffusion piloting schemes (to be discussed) serve to anchor the flame and ensure acceptable combustion efficiency and acoustic levels. The hot combustion gases are then accelerated to supersonic velocities in the nozzle. The flow continues to expand/accelerate through the diverging portion of the supersonic nozzle until it attains atmospheric conditions - ideal expansion.

Delivery of pilot fuel and cooling air is achieved through incorporation of centrifugal pumps attached to the sides of the engine. Air is delivered to the hot side of the rotor while pilot fuel is delivered to the cold or intake side. Each gas is collected in an annular manifold at the rotor's periphery and then directed to the required segments via internal segment manifolding.

The next sections will describe some of the experimental results of the pre-prototype engine located in Tacoma, WA.

\subsection{Experimental Results: The Pre-Prototype Engine}

\subsubsection{Experimental Set-up}

\subsubsection{Engine Layout}

Figure 3 illustrates the layout of the pre-prototype engine. The primary fuel employed was industrial grade methane (NG), supplied at a line pressure of 2 atmospheres and ambient temperature. The fuel system was manifolded and accurately measured by Worcester Model 20755X I/P valves and Sponsler Model SP1-1/2-CB-PH7-8-4RFX turbine meters. This base fuel was supplemented with pure hydrogen to assess alterations in the ignition delay times and flame speeds on combustor performance. Hydrogen was supplied by a $1200 \mathrm{~m} 3$ tube trailer, with an initial supply pressure of 136 atmospheres. Consistent with the methane supply, the hydrogen was regulated and controlled via I/P control valves and turbine meters. Downstream of the metering systems, the hydrogen and methane lines were joined and routed to the main engine fuel line. To eliminate any hydrogen backflow into the lower pressure methane line, a check valve was installed in the methane line.

Three fuel systems were installed in the engine. The first or primary fuel system was via radial fuel struts located inside the inlet air annulus (see Fig. 3). Due to their upstream location, fuel delivery struts induced a high level of mixedness by the time the mixture reached the rotor. The second or tertiary fuel system was via a series of porous metal plates circumferentially arranged around the inlet, downstream of the primary fuel struts. This also achieved a high degree of mixedness due to its upstream location. Both of the fuel systems supply the bulk of the gaseous fuel to the system and required only slightly above atmospheric pressures to operate effectively. The third or pilot fuel system supplied pure fuel to the flame stabilization device (to be discussed) and paralleled similar diffusion piloting schemes (Kendrick et al., 1999, Snyder et al., 1994). Injection of the pilot fuel was either through film-type slots located $1 \mathrm{~cm}$ upstream of the dump plane (interfacial plane dividing the end of the diffuser with the front of the combustor) 
and/or through the bluff body flame stabilizer itself. For all of the reported tests, only the diffusion piloting system was employed.

As with typical combustion systems, the engine was outfitted with a variety of instrumentation and diagnostic features to maximize output data from each combustion run. Combustor dynamic pressures and floor temperatures were measured using PCB Model 112A04 piezoelectric pressure transducers and a Land Infrared IR Thermometer Model X0144IRT, respectively. The IR detector viewed the combustor through a $1 \mathrm{~cm}$ diameter quartz window and ha a spectral range from 700-2000nm to accurately measure the liner floor temperature without significant contamination from such combustion byproducts as $\mathrm{CO} 2$ and $\mathrm{H} 2 \mathrm{O}$ (Eckbreth, 1988). Two additional IR detectors were mounted on the engine inlet and exhaust, and aligned with the axis of engine rotation, to monitor the combustor and nozzle strake wall temperatures. A $3 \mathrm{~cm}$ diameter quartz port was also added to the exhaust duct in order to view the broadband chemiluminescence via color video cameras.

The cavities formed between the rotor and the stationary case were purged with air (dried and filtered) to mitigate aerodynamic heating of the wheel space and prevent accumulation of flammable mixtures. This space was monitored for temperature changes (OMEGA type $\mathrm{K}$ thermocouples) and combustibles (Drager Multiwarn II catalytic).

Instrumentation interfaced with the System Control And Data Acquisition System (SCADA), and included emergency shut-off control logic where appropriate. All low speed data (gas flow rates, static pressures and temperatures) were monitored via RSView (Allen-Bradley), while control logic was managed by RSLogics software (Allen-Bradley). All high speed data (dynamic combustor pressures and liner temperatures) were similarly monitored in real time via a PC running a National Instruments WaveView7 system sampling up to $100 \mathrm{kHz}$ per channel. Post processing was facilitated via in-house Excel macros and documented in Excel workbooks.

Table 1: Engine Conditions (per ramjet).

\begin{tabular}{cccccc}
\hline $\begin{array}{c}\text { Mair } \\
(\mathrm{kg} / \mathrm{s})\end{array}$ & $\begin{array}{c}\text { Mfuel } \\
(\mathrm{kg} / \mathrm{s})\end{array}$ & $\begin{array}{c}\text { Pilot } \\
(\%, \text { by } \\
\text { volume })\end{array}$ & $\begin{array}{c}\text { Fuel } \\
-\end{array}$ & $\begin{array}{c}\mathrm{H} 2 \\
(\%, \text { by } \\
\text { volume })\end{array}$ & $\begin{array}{c}\text { AFC } \\
(\%, \text { mas } \\
\text { total flor }\end{array}$ \\
\hline 4 & $\leq .095$ & $0-100 \%$ & $\mathrm{NG} / \mathrm{H} 2$ & $0-70 \%$ & $10 \%$ \\
\hline
\end{tabular}

Table 1 details the range of flow conditions for a standard run. The "AFC" depicts the level of air film cooling (liner cooling) required to maintain acceptable combustor floor temperatures and hence mechanical system integrity. Engine starting and ramp up to design point speeds was accomplished with a $447 \mathrm{~kW}$ AC starter motor connected to a regenerative variable frequency drive unit (VFD). The VFD also compensates for ramjet thrust generation. The starting motor is required to "start" (Hertzberg et al., 1988) the inlet shock system to initiate positive thrust generation. Combustion was initiated at low rotor speeds via a pulse discharged igniter timed appropriately to the location of the dump plane via magnetic pick-ups. 


\subsubsection{Flame Stabilizers}

Three flame stabilizers were employed in this investigation (Figs. 5 and 6). The first is a $2.5 \mathrm{~cm}$ high, rearward facing step flameholder built into the engine's floor. The second builds from the first in that it employs vortex generators ("VG"s) $1 \mathrm{~cm}$ upstream of the step. This configuration was chosen to enhance mixing of the pilot fuel and core airflow and encourage a radial growth in combustion activity. Four sizes were investigated: 1, 1.3, 1.91 and $3.81 \mathrm{~cm}$ high. The final flame holder concept, "Pylon", was again an adaptation of the rearward facing step and was chosen to increase flame surface area through creation of multiple shear layers issuing from the dump plane.

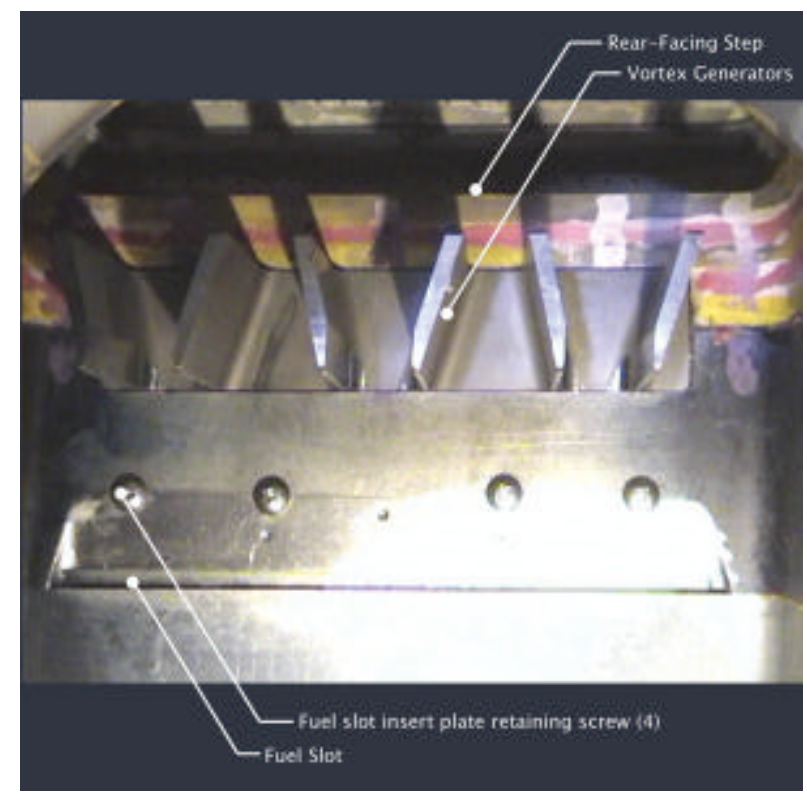

Fig. 5: Vortex Generator Flameholder.

\subsubsection{Experimental Results}

A timed spark from the Pulsed Discharge Igniters resulted in the simultaneous ignition of the combustor once pilot fuel had been initiated and engine speed had been stabilized at 800rpm. The fuel/oxidizer mixture ratio, ignition timing and AFC flow rates were optimized for greater than $95 \%$ ignition effectiveness irrespective of the flame stabilizer employed. 


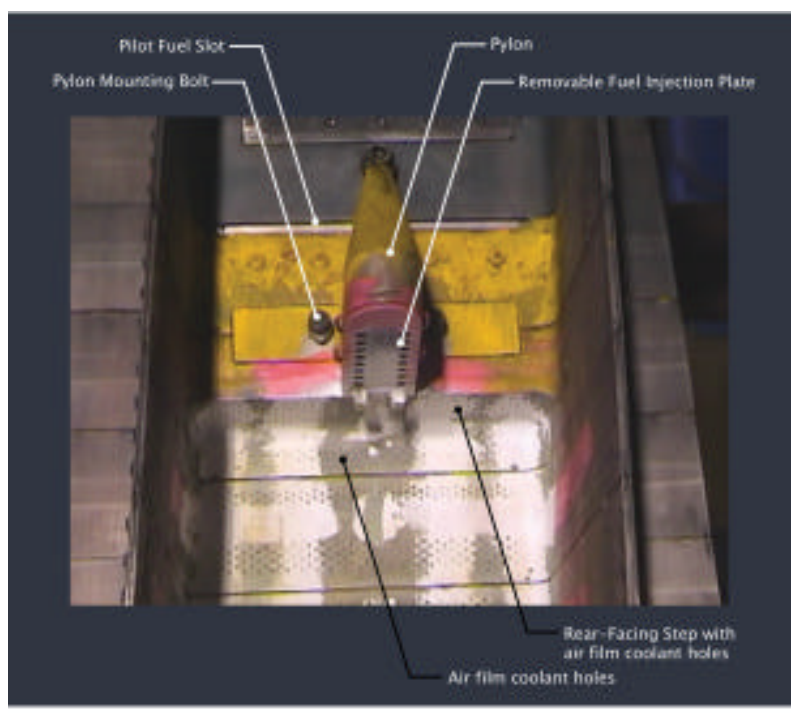

Fig. 6: Pylon and Rearward Facing Step.

Ignition was verified by monitoring the high speed data acquisition system and ensuring that both combustors revealed typical floor temperature traces as shown in Fig. 7 below. This particular test was conducted with the $1.9 \mathrm{~cm}$ tall VGs in place, located between the pilot fuel slot and the dump plane. Both combustors were lit as noted by the successive temperature pulses seen in the plot (top curve). It should be noted that due to the single location of the IR detector at the twelve-o-clock position on the engine case, adjacent pulses denote opposite combustors. Due to the sampling rate of the detector, the trace gives an accurate measure of the floor temperature as the rotor moves relative to the fixed position of the Land IR. In fact, taking into consideration the rotor's speed and sampling rate, the rotor travels approximately $3 \mathrm{~mm}$ between successive samples at the design speed (4300rpm). Also noted in the figure is the location of the beginning and end of the $33 \mathrm{~cm}$ long combustor as determined by magnetic pickups in the stationary engine case - see the square pulse (dump plane) and the triangle pulse (throat - middle curve). Also shown is the combustor pressure versus rotor position (bottom curve).

Evident is that the first half of the combustion chamber encompasses the recirculation zone and furthermore, that the location of the shear layer reattachment point is located approximately $20 \mathrm{~cm}$ downstream of the step (note the location of the maximum floor temperature). A relatively stationary thermal trace develops in the forward half of the combustor, fluctuating within $27 \mathrm{~K}$ from trace to trace. The latter half of the combustor, however, appears dominated by more incomplete combustion zones as evident by substantially greater temperature fluctuations $(+/-$ $120 \mathrm{~K})$ between successive cycles.

After stabilization at 800rpm for two minutes, an automated fuel, AFC and rotor ramp schedule was initiated via the Allen Bradley control logic (RSLogics) to attain the desired rim Mach number of approximately $1.1(4300 \mathrm{~mm})$. This inlet Mach number permits the exit nozzle to choke, and maximizes available thrust. In practice, the ramp (Fig. 8) is broken down into three separate ramps, which are individually optimized for adequate pilot fuel, AFC and rotor acceleration: 800-2000rpm, 2000-3000rpm and 3000-4300rpm. The main issue of using a 
single ramp for all the quantities was evident from the video and temperature traces during the ramping process. The fluid dynamics and combustion clearly alter as inlet/rotor speeds increase - most likely the combined effects of the non- uniform boundary conditions, increasing flow velocities, centrifugal effects and stratification with increasing centrifugal loadings. The AFC flow rates were found to be critical in ensuring a sufficiently robust flame during the ramping process. If not incremented correctly, it would eventually quench the entire combustion process.

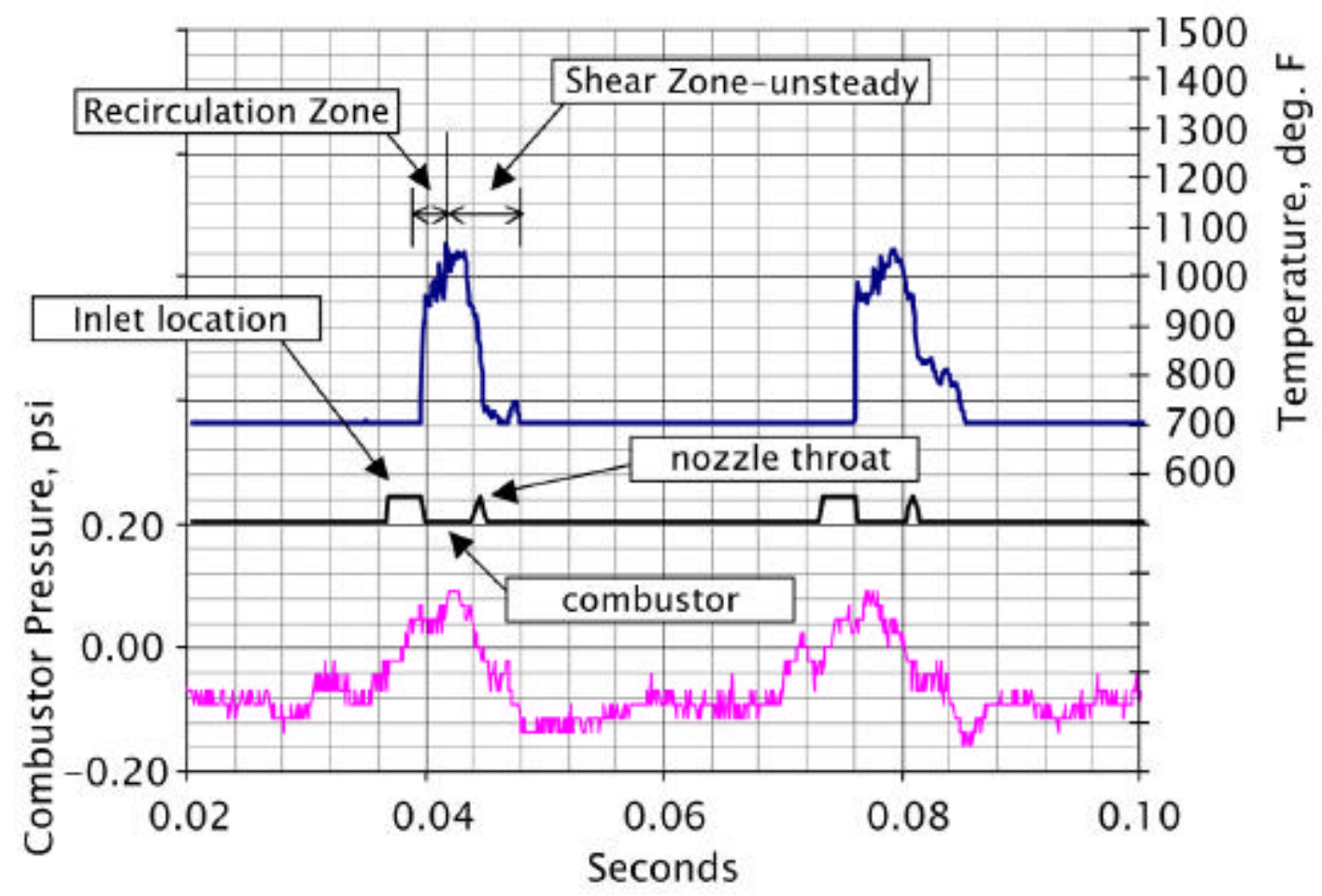

Fig. 7: Single rotor rotation showing combustor floor temperature and pressure traces.

Above 2000rpm, flame stabilization was generally restricted to the recirculation zone. The shear layer combustion, as indicated by the IR traces whose peaks move progressively upstream, was reduced as the overall combustor residence time dropped. Simple reactor modeling of the combustion zone (to be discussed) revealed that above 2000rpm, combustion is only possible in the recirculation zone where local residence times are conducive to methane oxidation. It appears, therefore, that there is a critical rotor speed above which the shear layer combustion is replaced by combustion within the recirculation zone.

The VFD is shown to hold the rotor at 4300rpm after some perturbations at the top of the ramp (Fig. 9). This transient is due to imbalance in required motor power transitioning from ramping to steady state operation. The power output required to maintain the engine's speed is a critical factor in the overall performance of the two thrust modules (combustor/nozzle systems). A 
typical test matrix (varying fuel loading between the three fuel circuits, $\mathrm{H} 2$ addition, etc.) is effectuated after the rotor speed and drive stabilizes.

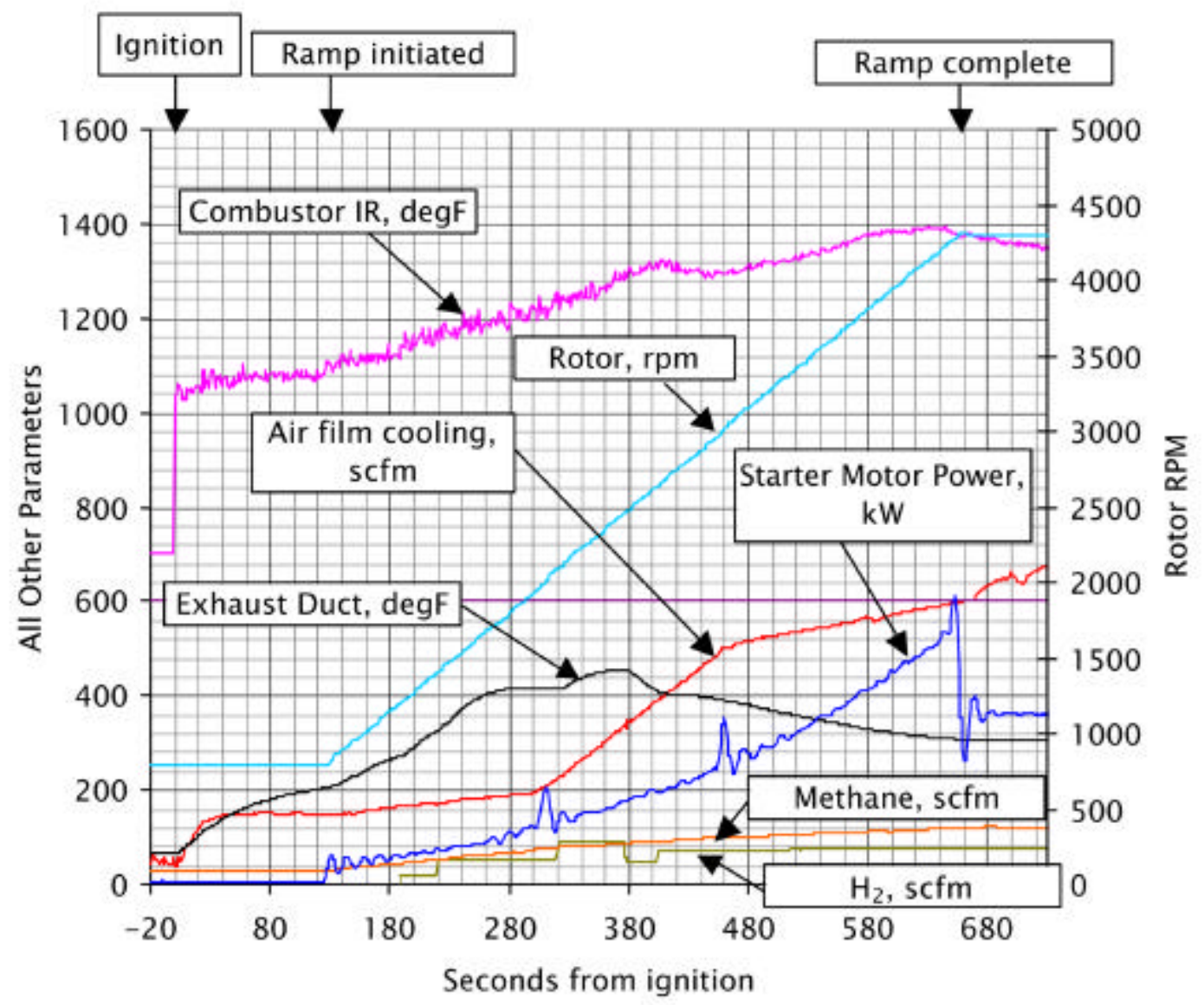

Fig. 8: Ramp Sequence to 4300rpm (Mach 1.1).

\subsubsection{Typical Test Sequence.}

Depicted in Table 2 is a sample test run whereby the methane flow rate was incrementally ramped to a prescribed point, where after the hydrogen level was then incremented. Also included on the table is the VFD power required to stabilize the engine rpm at 4300rpm. Decreases in the required VFD level is a direct measure of the effectiveness of the thrust modules and hence system efficiency. Points labeled 1 through 5 on the table refer to low speed data points (see Fig. 9) at prescribed operating conditions. Shown in Fig. 9 is a marked drop in the combustor floor temperature between points 1 and 3 . If it is assumed that the floor temperature is linked to the bulk average flame temperature, it would seem reasonable that the combustion zone is leaning out with this increase in NG flow rate and hence has passed through the stoichiometric fuel-air ratio. The argument can be extended through all points at a constant rotor speed of 4300rpm. Figure 10 depicts the indicated combustor floor temperature versus local equivalence ratio based on an estimate of the entrained airflow rate into the recirculation zone per the rationale above. The baseline air entrainment rate was determined by assuming that 
the maximum combustor temperature was coincident with stoichiometric conditions. The figure was then generated assuming the entrainment rate was preserved throughout the exercise.

Table 2: $1.3 \mathrm{~cm}$ Tall VGs Test Plan.

\begin{tabular}{cccccc}
\hline Pt. & $\begin{array}{c}\text { NG } \\
(\mathrm{Kg} / \mathrm{s})\end{array}$ & $\begin{array}{c}\mathrm{H} 2 \\
(\mathrm{Kg} / \mathrm{s})\end{array}$ & $\begin{array}{c}\text { VFD } \\
(\mathrm{KW})\end{array}$ & $\begin{array}{c}\mathrm{AFC} \\
(\mathrm{kg} / \mathrm{s})\end{array}$ & $\begin{array}{c}\mathrm{T} \\
(\mathrm{K})\end{array}$ \\
\hline & 0.041 & 0.0034 & 361 & 0.41 & 991 \\
& 0.054 & 0.0034 & 345 & 0.41 & 1020 \\
1 & 0.061 & 0.0034 & 342 & 0.41 & 1020 \\
& 0.071 & 0.0034 & 335 & 0.41 & 1014 \\
& 0.078 & 0.0034 & 335 & 0.41 & 1009 \\
2, & 0.084 & 0.0034 & 335 & 0.41 & 969 \\
3 & & & & & \\
4 & 0.081 & 0.0066 & 320 & 0.41 & 964 \\
& 0.071 & 0.0061 & 310 & 0.41 & 1011 \\
5 & 0.078 & 0.0061 & 310 & 0.41 & 989 \\
\hline
\end{tabular}

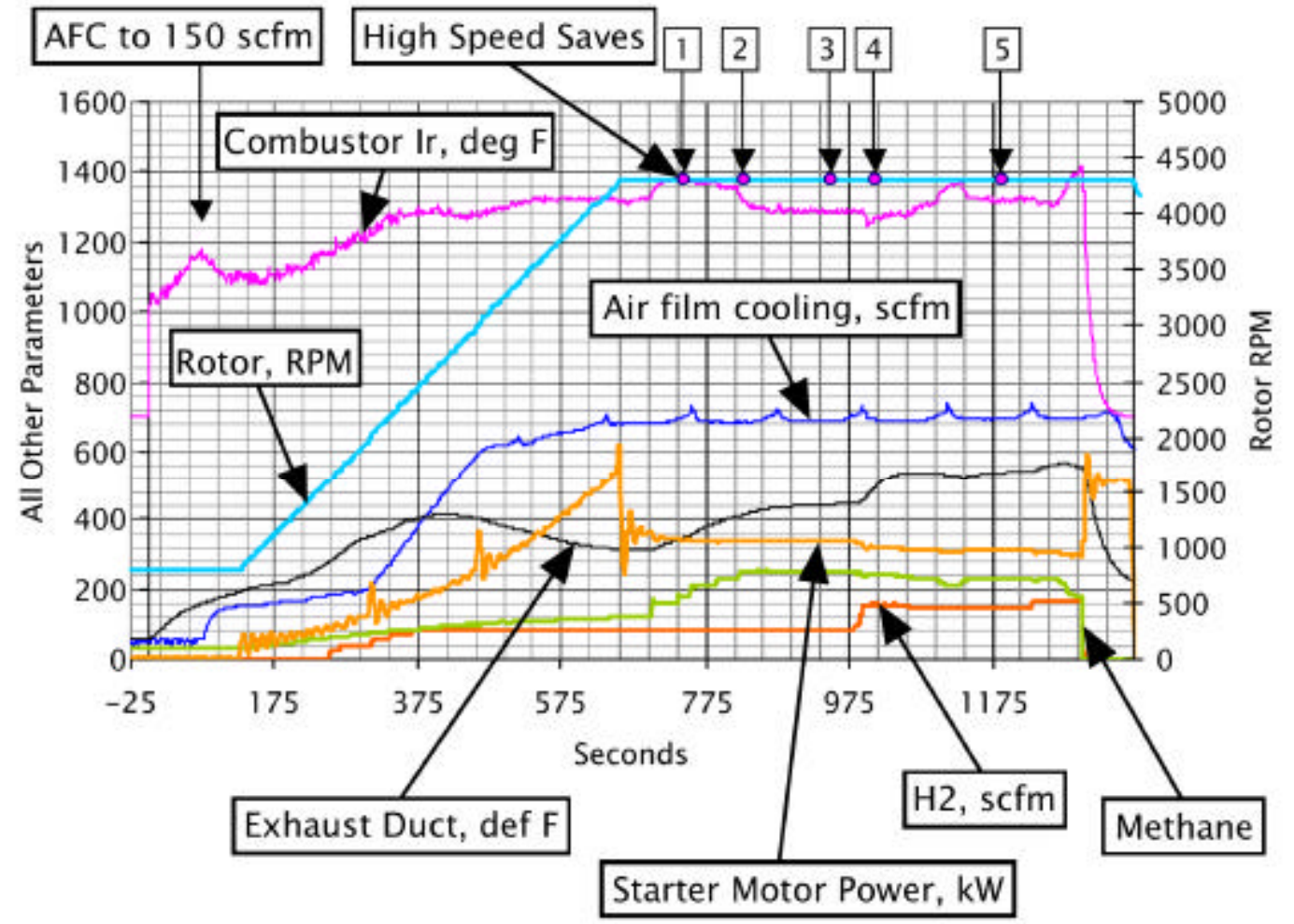

Fig. 9: NG and H2 Addition Test Plan with $1.3 \mathrm{~cm}$ VGs. 
Also shown in the figure are three curves estimating the floor temperature based on a simple, one dimensional model which includes the heat transfer from the gas to the floor (Kreith and Bohn, 1986) and equilibrium calculations (STANJAN) of flame temperatures for each fuel mixture. As demonstrated by the model, it is evident that the local equivalence ratio is a significant factor in determining the gas temperature.

Details of the above combustor are further explored through chemical reactor modeling of the events depicted in Fig. 9. It should be noted that at each of the denoted points, high speed data (IR and combustor pressure traces) were taken. A five element reactor network was used and is shown in Fig. 11. Two reactor blocks (\#1 and 2) were employed to model the shear layer which communicated with the recirculation zone (\#5). The shear layer half angle was assumed to be 5 degrees (Schlichting, 1979) and extended five duct heights downstream (Ozawa, 1981). The shear layer entrainment rate (as discussed above) was maintained at 3\% and all the fuel was assumed to enter element 1 to simulate the thin layer of fuel issuing from the fuel slot. The recirculation zone element received fuel through entrainment from neighboring elements 1 and 2. The GRI-MECH 2.12 was used to model the chemical kinetics mechanism for NG/H2 oxidation. It will be shown that the model accurately predicted the observed trends of Fig. 9.

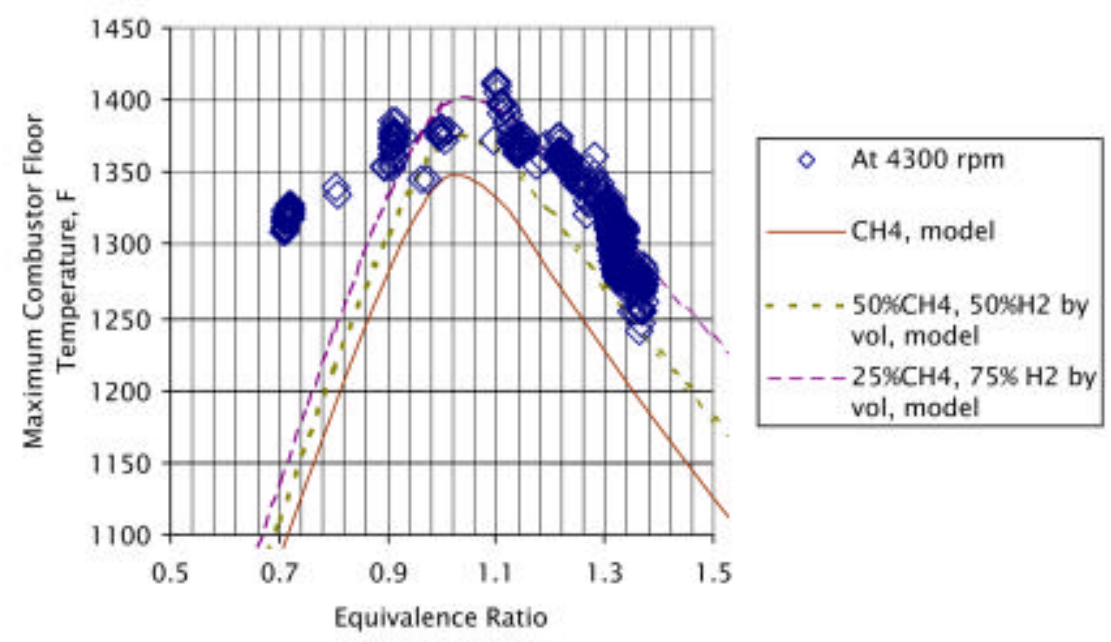

Fig. 10: Combustor Floor Temperatures versus Equivalence Ratio (data and model predictions).

Figure 12 shows the high speed data trace at point 1 for one rotation of the rotor. The top curve depicts the temperature while the bottom is the combustor pressure. Apparent is the forward location of the peak heat release within the combustor. A rapid drop ensues in the IR trace as the gas is unable to sustain combustion outside the recirculation zone.

Figure 13 depicts the modeling results corresponding to point 1. It reveals the predicted reactor temperatures based on the assumed flow rates and residence times determined experimentally. The highest temperature is seen to occupy reactor element $2(2908 \mathrm{~F})$ which corresponds to the shear layer near the middle and end of the recirculation zone, consistent with the high speed trace 
(Fig. 12). The recirculation zone (element 5) predicts a lower temperature (1303F), most likely attributed to the reduced fuel-air ratio and increased wall heat transfer at this location. Element 1 appears to be insensitive to local equivalence ratio and behaves more as a mixing element since its residence time is below the required blowoff residence time for its equivalence ratio $(?=$ 1.65). The temperature in the combustor is shown to drop off after the centrally located peak, most likely attributed to entrainment of the large, unreacted core flow and AFC flows with the products of combustion.
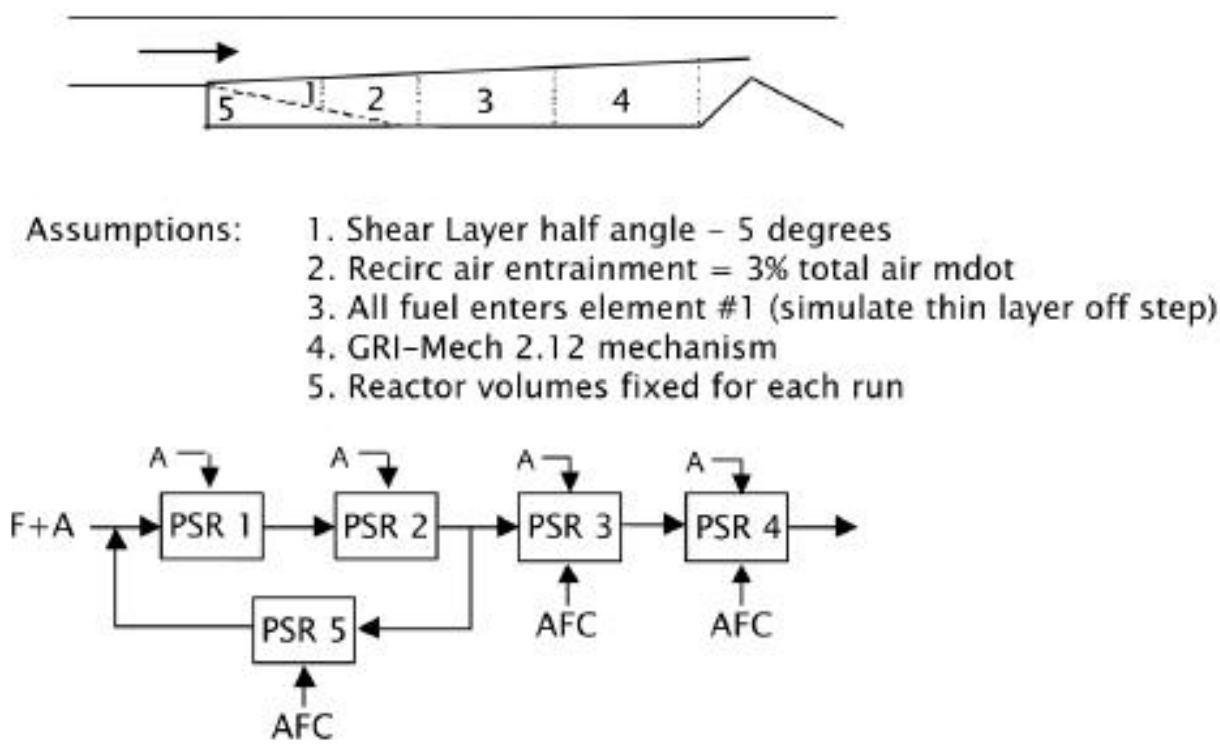

Fig. 11: Chemical Reactor Modeling Network.

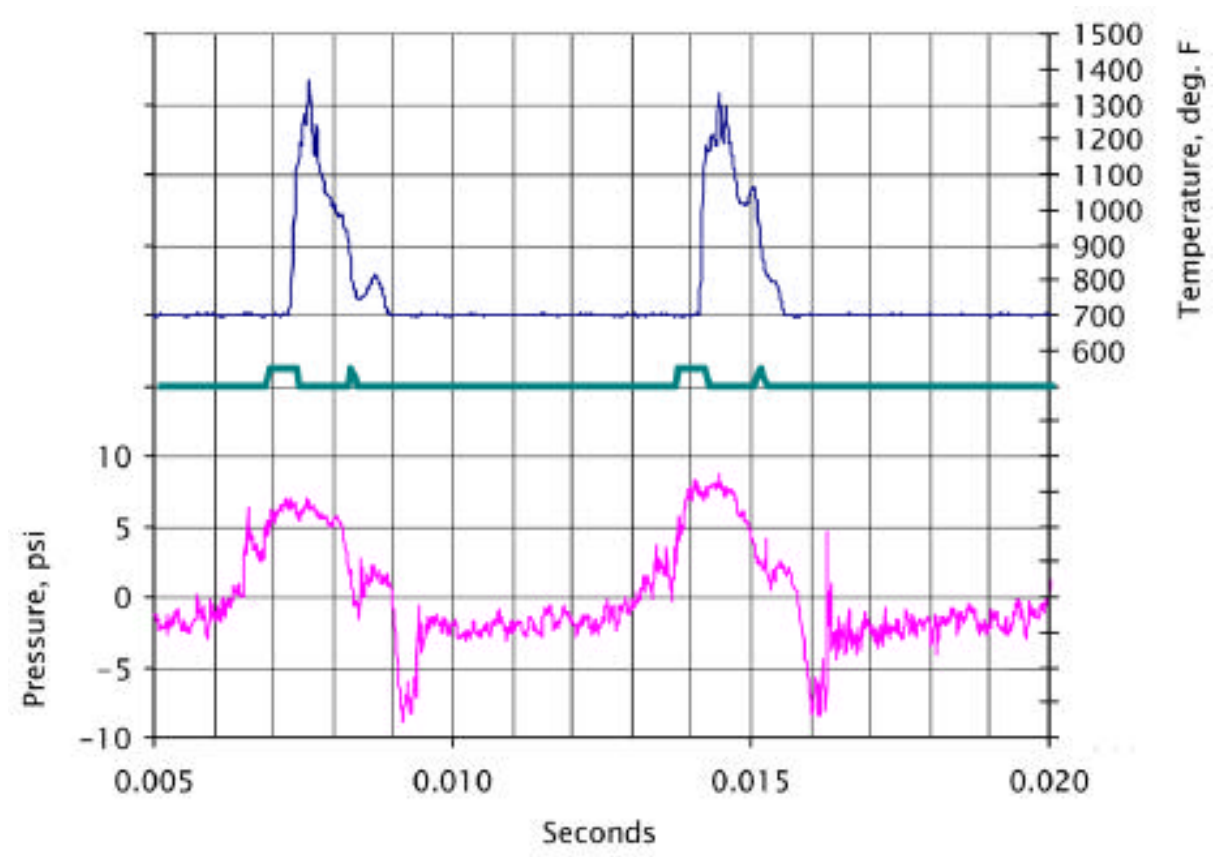

Fig. 12: High Speed Data Trace for Point 1. 
A similar analysis is made at point 2. The high speed data trace and reactor network modeling results are shown in Figs. 14 and 15, respectively. As evidenced in both figures, there is a downstream migration in the peak heat release location. The model results show that the fuel addition has quenched most combustion activity in the recirculation zone and shear layer and shifted it downstream. Residence times in the upstream elements (1,2 and 5) are below the required blowout times required to sustain combustion. The high speed data traces confirm these modeling results as evidenced by the combustion activity near the nozzle. Contrary to the model results is the reduced participation of the recirculation zone in the high speed data. This is most likely due to the model's over-prediction of fuel entering the recirculation zone.

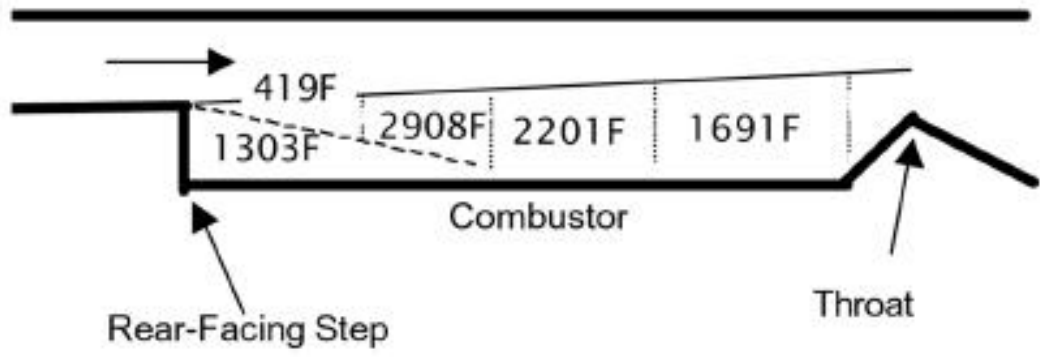

Fig. 13: Gas Temperature Modeling results for Point 1.

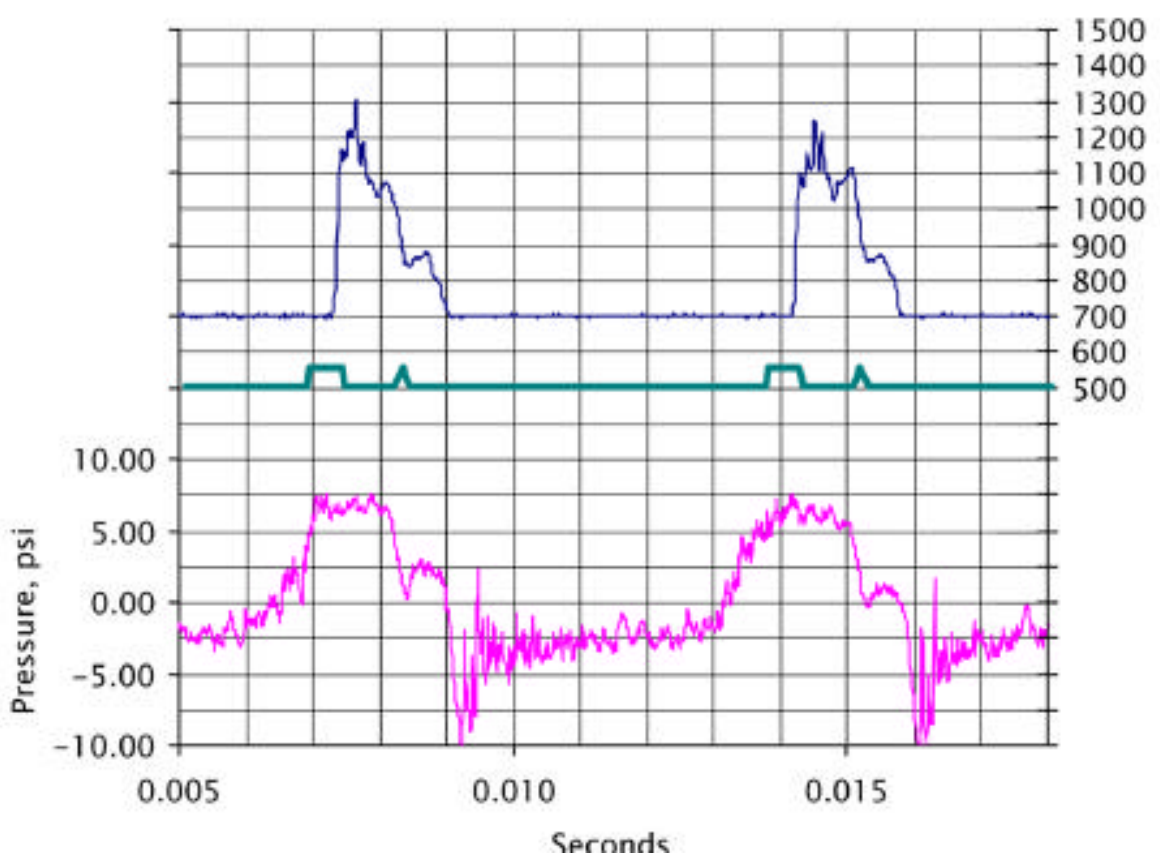

Fig. 14: High Speed Data Trace for Point 2. 
At the termination of the experiment, the maximum combustor floor temperature is $1413 \mathrm{~F}$. Combustion modeling results for this condition appear in Fig. 16 below. It appears that the recirculation zone residence times are adequate to sustain combustion with the hydrogen addition (compare Figs. 15 and 16) and such predictions are aligned with the temperature rise at the end of the test (Fig. 9).

\subsubsection{Comparisons in Flame Stabilization Configurations.}

As noted previously, three flame stabilization devices were tested: a baseline, rearward facing step, a rearward facing step and VGs, and a rearward facing step and Pylon. The mechanism for fuel-air mixing and flame stabilization with the baseline rearward facing step flameholder is conventional shear layer mixing and creation of a recirculation zone (Zukoski, 1978). The combustion is sustained by the steady entrainment of the combustion by-products into the shear layer, which ignites the incoming cold charge. The success of this flameholder, therefore, depends on the spreading rate of the shear layer, which must extend to the opposite wall to ensure high combustion efficiency. The inflow shear spreading half angle is approximately 5 degrees (Schlichting, 1979). This angle $(\phi)$ can be augmented though combustion (Glassman, 1987) by the following relation, where ST is the turbulent flame speed and $U$ is the approach velocity:

$$
\phi=\sin ^{-1}\left(\frac{S_{T}}{U}\right)
$$

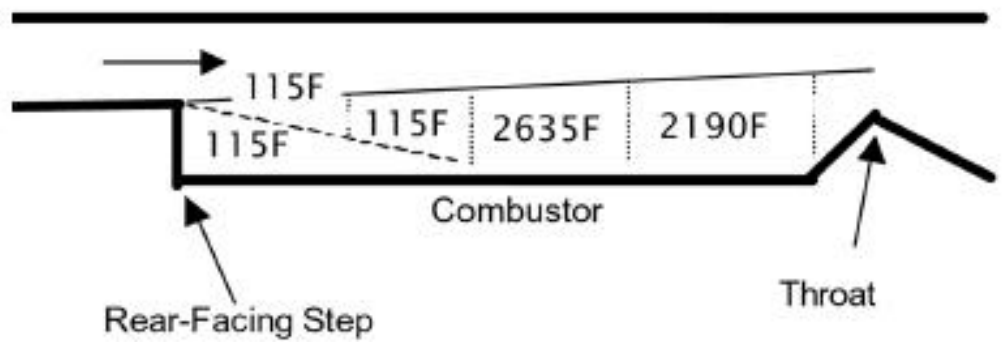

Fig. 15: Gas Temperature Modeling Results for Point 2.

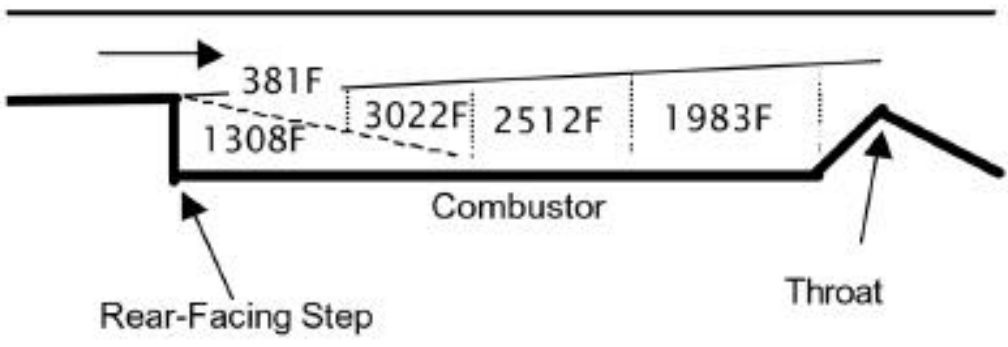

Fig. 16: Gas Temperature Modeling Results for Gas Off. 
Recognizing that the spreading angle can be augmented with a fuel with a higher turbulent flame speed, hydrogen was blended with the baseline methane fuel. Note that hydrogen's turbulent flame speed is up to four times methane's flame speed (Glassman, 1987). Tests with the baseline rearward facing step revealed poor mixing between the pilot fuel and mainstream airflow. As such, most of the pilot fuel directly entered the recirculation zone, resulting in over-rich conditions. These disadvantageous situations resulted in significant quenching of the combustion directly behind the step, and often shifted the combustion zone towards the centre of the combustion chamber where leaner conditions prevailed. Efforts were focused on enhancing the mixing of the pilot fuel stream with the main core flow with the implementation of the VGs.

Vortex Generators of various heights were examined with the intention of creating sufficient streamwise vorticity to enhance mixing of the pilot and core air flows to radially extend the combustion zone. Throughout the series of tests, it was found that there was a delicate balance between increased mixing (taller VGs) and reduced combustion stability. The larger the VGs, the less stable the combustion zone, most likely due to the increased turbulence and quenching activity promoted by the larger struts (Lefebvre, 1983). A sample high speed trace is shown in Fig. 17 for the optimal VG configuration (1 cm long).

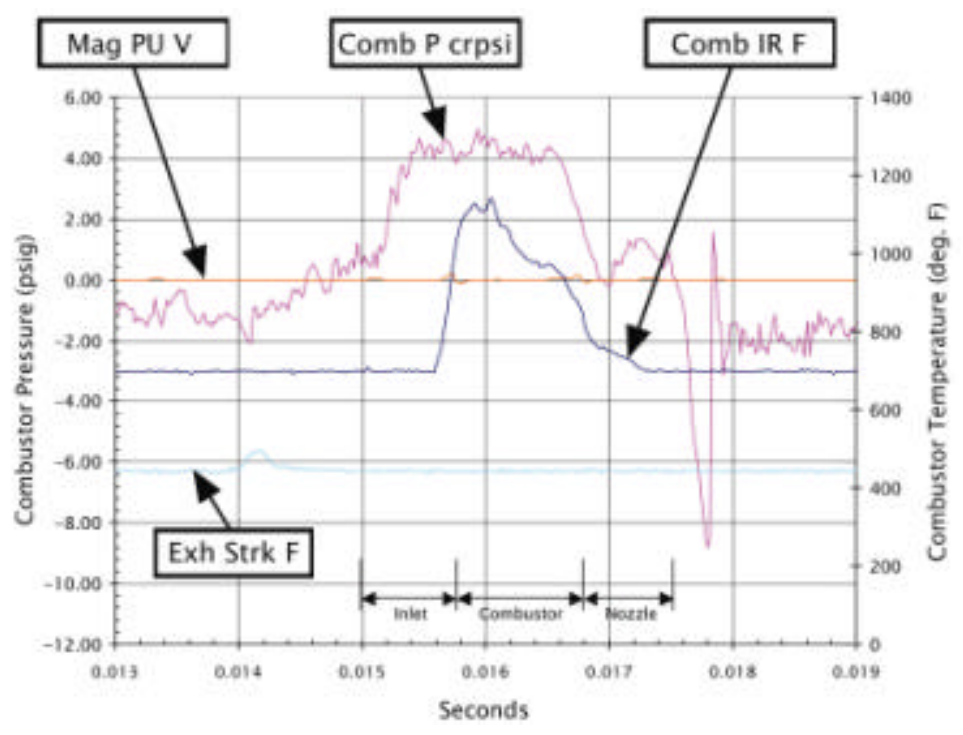

Fig. 17: High Speed trace for the $1 \mathrm{~cm}$ tall VGs.

Figure 17 shows that there is a greater streamwise distribution in temperature relative to the baseline step and secondly, the combustor pressure is more uniform. This is important since it is this pressure which forces the exhaust nozzle to choke, maximizing system performance.

The VGs provided some improvement with regards to heat release distribution and fuel-air mixing but failed to demonstrate a dramatic decrease in VFD power over the baseline configuration with fuel loading variations. The next series of tests investigated the Pylon design with various configurations in fuel injection (Fig. 6). This concept provides the greatest 
flexibility in fuel placement affords the combustor with the largest possible combustion volume to maximize heat release and provides the largest flame surface area. Such attributes favor an increase in overall combustion efficiency.

Preliminary CFD simulations using Star-CD showed encouraging results (Fig. 18). The pressure based solver was employed using the standard k-e turbulence model and included the effects of rotation. The figure depicts total temperature contours through various axial slices of the combustor. Apparent is the radial growth in combustion activity near the centre-line due to the presence of the Pylon. Unfortunately, due to the insufficient blockage afforded by the Pylon and the high momentum of the approach flow, it was ineffective in extending combustion in the axial direction. The design did, however, provide a more homogenous temperature distribution relative to the proceeding designs, irrespective of the fuel injection configuration (Fig. 19). This test was conducted with pilot fuel issuing from a single, $32 \mathrm{~mm}$ diameter hole from the pylon's faceplate and from the fuel slot along the floor. Due to this more uniform and elevated temperature distribution, combustion intensity and pressures were increased.
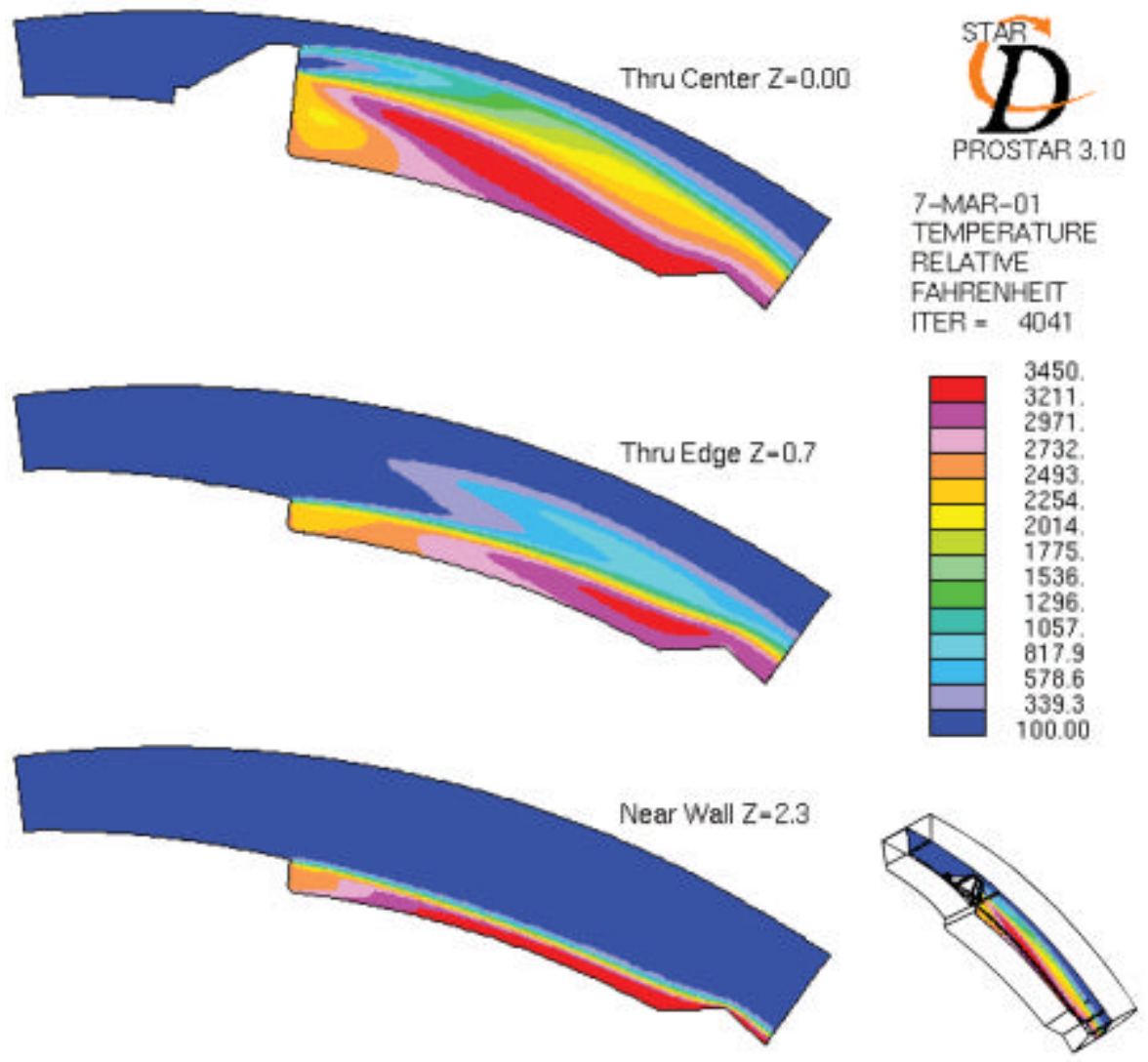

Fig. 18: Temperature Contours from a CFD Simulation of the Pylon Flame Stabilizer.

An overall comparison between VG and Pylon test data is made by comparing corresponding fuel loadings (BTUs of fuel) to VFD power for a rotor speed of 4300rpm. Lower VFD levels for the same fuel (BTU) loading would therefore signify higher system effectiveness. A summary 
plot appears in Fig. 20. All VG tests are denoted by square symbols while their Pylon counterparts are denoted by diamonds. Apparent is the more compact grouping of the Pylon data. The dotted box, which captures all such runs, further illustrates the fact that increasing fuel loading (expressed in BTU/s) lowers required VFD power. The greater slope and compactness of the Pylon data illustrates that it is more effective in flame holding, providing increased flame surface area and hence higher burner pressures through increased combustion activity. The net effect is higher ramjet thrust and overall cycle efficiency.

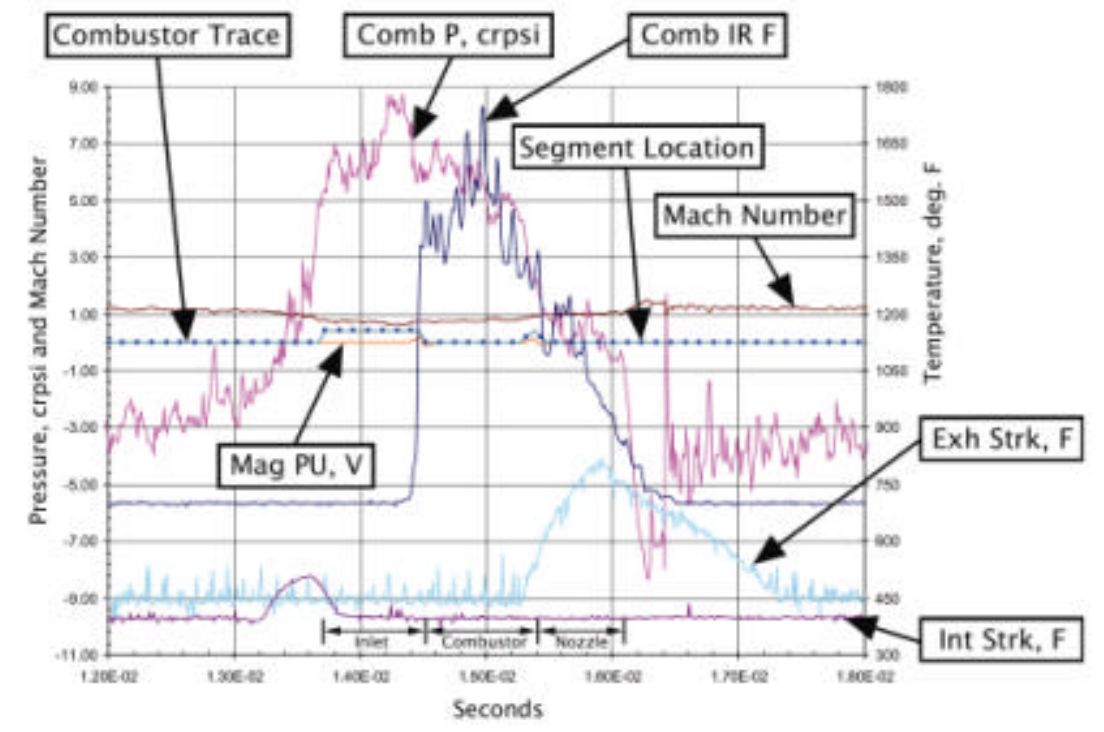

Fig. 19: High Speed for the Pylon Flame stabilizer with $32 \mathrm{~mm}$ fuel hole.

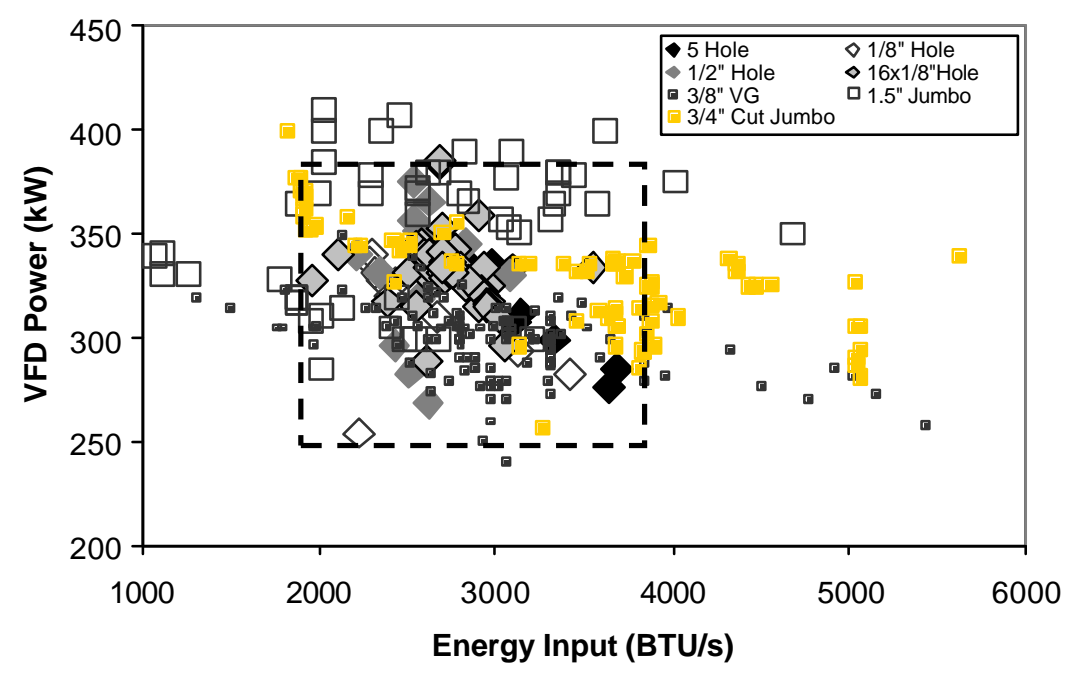

Fig. 20: VFD Comparisons between all VG and Pylon Runs. 


\subsection{Conclusions}

The preliminary results from a pre-prototype engine are presented. The engine is designed to convert the thrust from ramjet modules located on the rim of a disk into shaft torque, which in turn can be used for electrical power generation or mechanical drive applications. Initial combustion tests reveal the importance of flame volume and surface area for increased combustion efficiency, irrespective of fuel injection schemes. Chemical kinetic modeling was shown to accurately depict the essential flow features of a sample test matrix whereby stepwise increases in NG and hydrogen flow rates were separately investigated. The results confirm the visual observations that dramatic flowfield variations occur both during ramp up and pilot fuel excursions due to the nature of the engine. 


\subsection{REFERENCES}

4 Bogdanoff, D. W., 1992, "Ram Accelerator Direct Space Launch System: New Concepts," J. Prop. Pwr., Vol. 8, No. 2, pp. 481-490.

5 Eckbreth, A. C., 1988, Laser Diagnostics for Combustion Temperature and Species, Turnbridge Wells, Kent

6 Glassman, I., 1987, Combustion, Academic Press, Orlando.

7 Harsha, P. T., and Edelman, R. B., 1981, "Assessment of a Modular Ramjet Combustion Model," J. Spacecraft, Vol. 19, No. 3, pp. 430-436.

8 Heiser, W. H. and Pratt, D. T., 1994, Hypersonic Airbreathing Propulsion, AIAA Ed. Series, Washington.

9 Hertzberg, A., Bruckner, A. P. and Bogdanoff, D. W., 1988, "Ram Accelerator: A New Chemical Method for Accelerating Projectiles to Ultrahigh Velocities," AIAA Journal, Vol. 26, No. 2, pp. 195-203.

10 Kendrick, D. W., Anderson, T. J., Sowa, W. A., and Snyder, T. S., 1999, "Acoustic Sensitivities of Lean-Premixed Fuel Injectors in a Single Nozzle Rig," J. of Eng. For Gas Turb. And Pwr, Vol. 121, pp. 429-436.

11 Kreith, F. and Bohn, M. S., 1986, The Principals of Heat Transfer, Harper and Row, Cambridge.

12 Lefebvre, A. H., 1983, "Gas Turbine Combustion”, Hemisphere Publishing,

13 Oates, G. C., 1997, Aerothermodynamics of Gas Turbine and Rocket Propulsion, AIAA Ed. Series, Washington.

14 Ozawa, R.I., 1981, "Survey of Basic Data on Flame Stabilization and Propagation for High Speed Combustion Systems", AFAPL-TR-70-81.

15 Schlichting, H., 1979, Boundary-Layer Theory, McGraw-Hill, New York.

16 Snyder, T. S., Rosfjord, T. J., McVey, J., Hu, A., and Schlein, B., 1994, "Emissions and Performance of a Lean-Premixed GasFuel Injection System for Aeroderivative Gas Turbine Engines," ASME Paper 94-GT-234.

17 Yungster, S., Eberhardt, S. and Bruckner, A. P., 1991, "Numerical Simulation of Hypervelocity Projectiles in Detonable Gases," AIAA Journal, Vol. 29, No. 2, pp. 187-193.

18 Zukoski, E.E., 1978, "Afterburners," The Aerothermodynamics of Aircraft Gas Turbine Engines, AFAPL-TR-78-52, Ch. 21. 